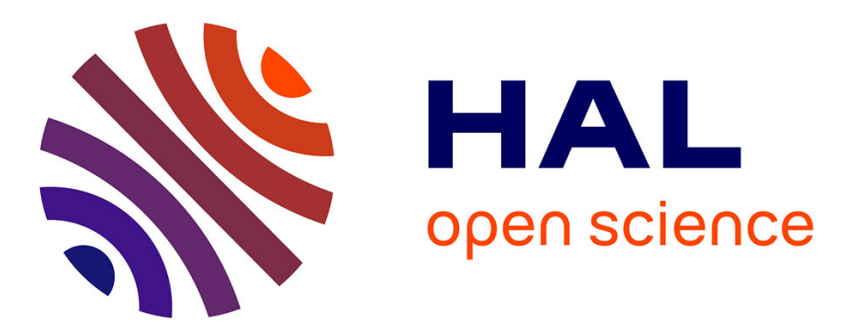

\title{
Two-flux transfer matrix model for predicting the reflectance and transmittance of duplex halftone prints
}

Serge Mazauric, Mathieu Hébert, Lionel Simonot, Thierry Fournel

\section{To cite this version:}

Serge Mazauric, Mathieu Hébert, Lionel Simonot, Thierry Fournel. Two-flux transfer matrix model for predicting the reflectance and transmittance of duplex halftone prints. Journal of the Optical Society of America, 2014, 31, pp.2775. 10.1364/JOSAA.31.002775 . hal-01088864

\section{HAL Id: hal-01088864 https://hal.science/hal-01088864}

Submitted on 28 Nov 2014

HAL is a multi-disciplinary open access archive for the deposit and dissemination of scientific research documents, whether they are published or not. The documents may come from teaching and research institutions in France or abroad, or from public or private research centers.
L'archive ouverte pluridisciplinaire HAL, est destinée au dépôt et à la diffusion de documents scientifiques de niveau recherche, publiés ou non, émanant des établissements d'enseignement et de recherche français ou étrangers, des laboratoires publics ou privés. 


\title{
Two-flux transfer matrix model for predicting the reflectance and transmittance of duplex halftone prints
}

\author{
Serge Mazauric, ${ }^{1,2, *}$ Mathieu Hébert, ${ }^{1}$ Lionel Simonot, ${ }^{3}$ and Thierry Fournel $^{1}$ \\ ${ }^{1}$ Université de Lyon, Université Jean Monnet de Saint-Etienne, CNRS UMR5516 Laboratoire Hubert Curien, \\ F-42000 Saint-Etienne, France \\ ${ }^{2}$ CPE Lyon, Domaine Scientifique de la Doua, 43 boulevard du 11 Novembre 1918 BP 82077, 69616 Villeurbanne Cedex, \\ France \\ ${ }^{3}$ Université de Poitiers, CNRS UPR 3346 Institut Pprime, 11 Boulevard Marie et Pierre Curie, BP 30179, \\ F-86962 Futuroscope Chasseneuil Cedex, France \\ ${ }^{*}$ Corresponding author: serge.mazauric@univ-st-etienne.fr
}

Received July 25, 2014; revised October 24, 2014; accepted October 24, 2014; posted October 28, 2014 (Doc. ID 218859); published November 21, 2014

\begin{abstract}
We introduce a model allowing convenient calculation of the spectral reflectance and transmittance of duplex prints. It is based on flux transfer matrices and enables retrieving classical Kubelka-Munk formulas, as well as extended formulas for nonsymmetric layers. By making different assumptions on the flux transfers, we obtain two predictive models for the duplex halftone prints: the "duplex Clapper-Yule model," which is an extension of the classical Clapper-Yule model, and the "duplex primary reflectance-transmittance model." The two models can be calibrated from either reflectance or transmittance measurements; only the second model can be calibrated from both measurements, thus giving optimal accuracy for both reflectance and transmittance predictions. The conceptual differences between the two models are deeply analyzed, as well as their advantages and drawbacks in terms of calibration. According to the test carried out in this study with paper printed in inkjet, their predictive performances are good provided appropriate calibration options are selected. @ 2014 Optical Society of America OCIS codes: (000.3860) Mathematical methods in physics; (100.2810) Halftone image reproduction;

(120.5700) Reflection; (120.7000) Transmission; (230.4170) Multilayers.
\end{abstract} http://dx.doi.org/10.1364/JOSAA.31.002775

\section{INTRODUCTION}

For a long time, the variation of the spectral properties of surfaces and objects by application of coatings has been a wide subject of investigation for physicists, who have proposed several models based on specific mathematical formalisms according to the type of physical components and the application domain. In the domain of paints, papers, and other diffusing media, a classical approach is to use the Kubelka-Munk system of two coupled differential equations to describe the propagation of diffuse fluxes in the medium [1,2]. The extension of this model by Kubelka to stacks of paint layers is based on geometrical series describing the multiple reflections and transmissions of these diffuse fluxes between the different layers [3,4]. Geometrical series were also used by Saunderson [5] when deriving his correction of the Kubelka-Munk model in order to account for the internal reflections of light between the paint layer and the paint-air interface, by Clapper and Yule [6] in their reflectance model for halftone prints to account for the internal reflections of light between the paper and the print-air interface across the inks, and by Williams and Clapper [7] in their model for gelatin photograph to account for the internal reflections of light between the paper and the printair interface across the nonscattering gelatin layer. More recently, alternative mathematical methods using graphs [ㅁ] Markov chains [9], or continuous fractions [10,11] were proposed to derive the equations of these models in a more efficient way, especially when their structure or the number of layers increases. Despite their apparent dissimilarity, all these models have in common to be comparable to a two-flux model describing the mutual exchanges between downward and upward propagating light quantities, by reflection or transmission of light by the different layers and surfaces. In the case of weakly scattering media, e.g., pigmented media $[\underline{12}, \underline{13}]$, advanced models based on the radiative transfer theory [14], on multiflux approaches [15], or on a probabilistic description of photon paths [ㅌ-19], are needed to take into account more thoroughly the orientations of scattered light. However, in all the previously cited configurations where the media are either very scattering or almost nonscattering, the two-flux-like approach generally provides good prediction accuracy and the equations can be turned into vector equations involving $2 \times 2$ transfer matrices. This matrix formalism is well known in the case of thin films illuminated by coherent light modeled by complex amplitudes of electromagnetic fields [20], or in the case of diffusing layers illuminated by diffuse incoherent fluxes [21], but it is less known that similar formalism actually applies to any stack of layers [22], provided appropriate light models (oriented collimated fluxes or diffuse fluxes) are used for each type of layer. In comparison to more classical mathematical methods, the matrix method, presented in Section 2 , may considerably ease the derivation of analytical expressions for the reflectance and 
transmittance of multilayer specimens; it also enables direct numerical computation. In this paper, we especially use it to predict the reflectance and transmittance of duplex halftone prints.

The two-flux transfer matrix approach has a natural link with the Kubelka-Munk model, noticed in various previous studies [10, 22, 23]. Since transfer matrices allow addressing nonsymmetrical components, i.e., layers or stack of layers having different reflectance on their two sides, we can easily generalize the Kubelka-Munk model to nonsymmetric components; we therefore saw some interest in summarizing this approach in Section 3 and Appendices A and B, although it is not directly used in our model for duplex halftone prints. However, our model includes the so-called Saunderson correction that takes into account the flux transfers at the bordering interfaces of a diffusing layer, thanks to a transfer matrix formalism comparable to the one introduced by Emmel in [23] but extended to a larger variety of illumination-observation geometries (Section 4).

Transmittance of halftone prints is generally much less analyzed than reflectance, mainly because the printing support is often opaque. However, various kinds of prints are illuminated from the back side, e.g., light billboards at night (which are illuminated on the front face during the day). There is some interest in predicting both spectral reflectance and transmittance for these prints. Moreover, not much effort is needed to extend the predictive model to duplex prints, i.e., papers printed on both faces with different color images. In order to predict the reflectance and transmittance of duplex halftone prints, we introduce in Sections 5 and 6 a "duplex primary reflectance-transmittance (DPRT)" model, which is sensibly different from the previously proposed model inspired of the Williams-Clapper and Clapper-Yule models where the ink layers are assumed to be nonscattering $[9,24]$. In order to compare more easily the two models, we review the Clapper-Yule-inspired model, called "duplex Clapper-Yule (DCY)" model, with the transfer matrix formalism in Section 7. In the DPRT model, no assumption is made regarding the scattering properties of the inks. Despite its calibration needs more measurements than the DCY model, its main interest lies in the fact that the calibration of its parameters can combine reflectance and transmittance measurements, whereas the DCY model is calibrated either from reflectance or transmittance measurements. Consequently, the DPRT model needs one set of parameters to accurately predict the reflectance and transmittance of a duplex halftone print, while the DCY models needs two sets of parameters, one being optimal for the reflectance prediction, the other one being optimal for the transmittance prediction. These conceptual differences are explained in Section $\underline{8}$ and illustrated through experiments carried out with samples printed in inkjet. Finally, our conclusions are drawn in Section $\underline{9}$.

\section{TWO-FLUX TRANSFER MATRIX MODEL}

The matrix transfer model applies with a stack of planar optical components such as layers of materials or interfaces between layers. These components are said to be symmetric when they have same reflectance (and same transmittance) on their two faces: this is often the case for layers of typical homogenous materials used in color reproduction (paint, paper, inks). However, many components may be nonsymmetric, for example because they are themselves a superposition of different components. Interfaces are nonsymmetric components too. We therefore characterize every component by four transfer factors: the front-side reflectance $r$, the back-side reflectance $r^{\prime}$, the forward transmittance $t$, and the backward transmittance $t$ '.

In a stack of components, the fluxes propagating forward and backward are respectively denoted $I_{k}$ and $J_{k}$, where $k$ indicates the position in the stack (see Fig. 1). Note that all fluxes and transfer factors in this model may depend upon wavelength.

In each component $k$, one can write the following relations between incoming and outgoing fluxes:

$$
J_{k-1}=r_{k} I_{k-1}+t_{k}^{\prime} J_{k}, I_{k}=t_{k} I_{k-1}+r_{k}^{\prime} J_{k},
$$

and turn them into the following vector equation:

$$
\left(\begin{array}{cc}
-r_{k} & 1 \\
t_{k} & 0
\end{array}\right)\left(\begin{array}{c}
I_{k-1} \\
J_{k-1}
\end{array}\right)=\left(\begin{array}{cc}
0 & t_{k}^{\prime} \\
1 & -r_{k}^{\prime}
\end{array}\right)\left(\begin{array}{c}
I_{k} \\
J_{k}
\end{array}\right),
$$

or, assuming $t_{k} \neq 0$,

$$
\left(\begin{array}{c}
I_{k-1} \\
J_{k-1}
\end{array}\right)=\frac{1}{t_{k}}\left(\begin{array}{cc}
0 & 1 \\
t_{k} & r_{k}
\end{array}\right)\left(\begin{array}{cc}
0 & t_{k}^{\prime} \\
1 & -r_{k}^{\prime}
\end{array}\right)\left(\begin{array}{c}
I_{k} \\
J_{k}
\end{array}\right),
$$

which yields

$$
\left(\begin{array}{c}
I_{k-1} \\
J_{k-1}
\end{array}\right)=\mathbf{M}_{k}\left(\begin{array}{c}
I_{k} \\
J_{k}
\end{array}\right)
$$

where $\mathbf{M}_{k}$ is the transfer matrix representing the component $k$ :

$$
\mathbf{M}_{k}=\frac{1}{t_{k}}\left(\begin{array}{cc}
1 & -r_{k}^{\prime} \\
r_{k} & t_{k} t_{k}^{\prime}-r_{k} r_{k}^{\prime}
\end{array}\right)
$$

Regarding the two components 1 and 2 together, Eq. (4) can be repeated twice and one gets

$$
\left(\begin{array}{l}
I_{0} \\
J_{0}
\end{array}\right)=\mathbf{M}_{1}\left(\begin{array}{l}
I_{1} \\
J_{1}
\end{array}\right)=\mathbf{M}_{1} \mathbf{M}_{2}\left(\begin{array}{l}
I_{2} \\
J_{2}
\end{array}\right)=\mathbf{M}\left(\begin{array}{c}
I_{2} \\
J_{2}
\end{array}\right),
$$

where $\mathbf{M}$ is the transfer matrix representing the two layers together, similarly defined as Eq. (ㄷ) in terms of its transfer factors $r, t, r^{\prime}$, and $t^{\prime}$.

Equation (6) shows that the transfer matrix representing two superposed components is the product of the component's individual transfer matrices. The multiplicativity of transfer matrices is true for any number of components,

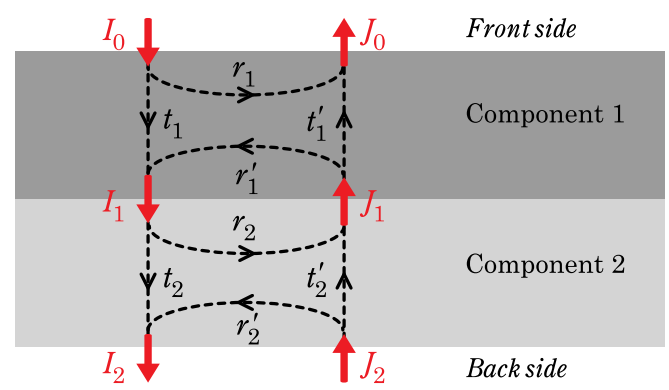

Fig. 1. Flux transfers between two flat components (the arrows do not render orientation of light). 
and the left-to-right position of the matrices in the product reproduces the front-to-back position of the corresponding components. Every transfer matrix in this model has the structure displayed in Eq. (5) and from a given transfer matrix $\mathbf{M}=\left\{m_{i j}\right\}$, provided $m_{11} \neq 0$, one retrieves the transfer factors in the following way:

$$
\begin{gathered}
r=m_{21} / m_{11}, \\
t=1 / m_{11}, \\
r^{\prime}=-m_{12} / m_{11}, \\
t^{\prime}=\operatorname{det} \mathbf{M} / m_{11}=m_{22}-m_{21} m_{12} / m_{11} .
\end{gathered}
$$

Similar transfer matrix models are used in the domain of thin films for coherent or partly coherent directional light reflected between the interfaces of a transparent substrate or a thin film, or a pile of plates [20,25-28], where reflectances and transmittances are replaced with Fresnel reflectivities and transmittivities (ratios of the electric field amplitudes [29]). A model based on diffuse fluxes is also presented in the appendix of [22].

\section{STACKS OF DIFFUSING LAYERS}

Applying formulas (7)-(10) to the matrix $\mathbf{M}_{1} \cdot \mathbf{M}_{2}$ representing the stack of layers displayed in Fig. 1 yields

$$
\begin{aligned}
r & =r_{1}+\frac{t_{1} t_{1}^{\prime} r_{2}}{1-r_{1}^{\prime} r_{2}}, & t & =\frac{t_{1} t_{2}}{1-r_{1}^{\prime} r_{2}}, \\
r^{\prime} & =r_{2}^{\prime}+\frac{t_{2} t_{2}^{\prime} r_{1}^{\prime}}{1-r_{1}^{\prime} r_{2}}, & t^{\prime} & =\frac{t_{1}^{\prime} t_{2}^{\prime}}{1-r_{1}^{\prime} r_{2}} .
\end{aligned}
$$

Kubelka, in his study on nonhomogenous diffusing media [3], obtained the same equations where the flux transfers correspond to the diffuse reflectances and transmittances of strongly scattering layers. The method he used, i.e., the description of all possible flux paths yielding geometrical series, is feasible with two superposed components, even three, but not more. For a stack of many components, similar equations can be used iteratively, first with components 1 and 2 , then with components $1+2$ and 3 , then with components $1+2+$ 3 and 4 and so on. In contrast, the matrix method considers all components simultaneously, and the flux transfers between them are described in one matrix equation.

Obviously, the analytical expressions for the transfer factors of a stack become more complex as the number of components increases, except when all the components are identical: a stack of $x$ components, individually represented by matrix $\mathbf{M}$, is represented by the matrix $\mathbf{M}^{x}$, which is easily calculated through the diagonalization of $\mathbf{M}$. This calculation, presented in [10] in the case of symmetrical diffusing layers and extended in Appendix A for nonsymmetric components, yields close-form reflectance and transmittance formulas as functions of the number of components. By considering the stack of components as an effective, homogenous layer, the matrix $\mathbf{M}^{h}$ gives the upward and downward fluxes at any altitude $h$ in the stack, where $h$ may even be a real number.

Except specific applications in color reproduction, such as the stacking of printed films presented in [11], the interest of these analytical formulas is especially that they enable retrieving the Kubelka-Munk hyperbolic reflectance and transmittances formulas [2] by decomposing a homogenous layer into an infinity of identical sublayers. The demonstration was also given in [10] for symmetrical scattering layers. In Appendix $\underline{B}$, we review it by keeping the nonsymmetry of the matrices, i.e., by considering different scattering and absorption coefficients for upward and backward fluxes. This original idea was directly inspired by the facility do deal with nonsymmetrical components with the matrix approach.

\section{NONSYMMETRIC DIFFUSING LAYER WITH INTERFACES}

The transfer matrix representing a strongly scattering, possibly nonsymmetric layer is similar to Eq. (ㅁ) where the transfer factors are the diffuse reflectances $\rho$ and $\rho^{\prime}$ and transmittances $\tau$ and $\tau^{\prime}$ of the layer:

$$
\mathbf{M}=\frac{1}{\tau}\left(\begin{array}{cc}
1 & -\rho^{\prime} \\
\rho & \tau \tau^{\prime}-\rho \rho^{\prime}
\end{array}\right) .
$$

Since the layer is generally surrounded by a medium with different refractive index, e.g., air, there are also flux transfers at its bordering interfaces. In the context of the Kubelka-Munk model, which models only the reflectance and transmittance of the diffusing layer without interfaces, accounting the flux transfers at the interface with air is known as the "Saunderson correction." The correcting formula proposed by Saunderson considers one interface with air, illumination at $45^{\circ}$, and observation in the normal of the surface [5]. This "correction" may be extended to other measuring geometries, and can be applied to the two bordering interfaces of a layer. Here, we propose to review it in a general manner using the transfer matrix model.

The flux transfers by an interface are derived from the Fresnel formulas according to the angular distribution of light at both sides of the interface. We can recall that when directional light incomes from air (respectively from the medium) at angle $\theta$, the angular reflectance, denoted $R_{01}(\theta)$ [respectively $R_{10}(\theta)$ ], is given by the Fresnel formulae [29]. The air-tomedium and medium-to-air transmittances are respectively $T_{01}(\theta)=1-R_{01}(\theta)$ and $T_{10}(\theta)=1-R_{10}(\theta)$. When the incident light at the air side is perfectly diffuse (i.e., Lambertian), the reflectance of the interface is denoted as $r_{01}$ according to the notations introduced in [30], and obtained by integrating the Fresnel angular reflectance $R_{01}(\theta)$ over the hemisphere as follows [31]:

$$
r_{01}=\int_{\theta=0}^{\pi / 2} R_{01}(\theta) \sin (2 \theta) \mathrm{d} \theta
$$

The diffuse air-to-medium transmittance is $t_{01}=1-r_{01}$, the diffuse reflectance at the medium side is $r_{10}=1-\left(1-r_{01}\right) /$ $n^{2}$, and the diffuse medium-to-air transmittance is $t_{10}=$ $t_{01} / n^{2}$, where $n$ is the refractive index of the medium [24].

The reflectance of the interface at the air side is denoted as $r_{s}$ (see Fig. 2); it may be zero if the detector is out of the specular direction. The air-to-medium transmittance, $t_{\text {in }}$, depends only on the illumination geometry and the medium-to-air transmittance, $t_{\text {out }}$, depends only on the observation geometry. Since the measuring geometry is important for accurate 


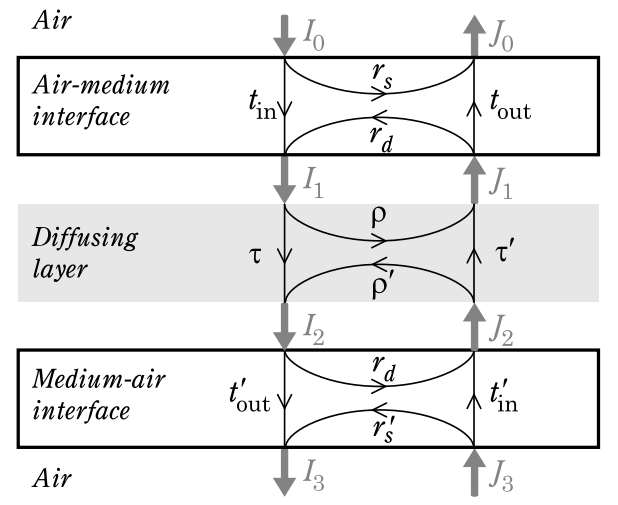

Fig. 2. Flux transfer between a diffusing layer and its interfaces with air.

prediction by the model, we recall in Table 1 the expressions of $r_{s}, t_{\text {in }}$, and $t_{\text {out }}$ for typical measuring geometries [32], denoted under the form " $x y: z$ " according to the notation recommended by the CIE [33] where $x$ denotes the incident geometry ("d" for Lambertian incident light, or an angle in degree for directional incident light in a specific angle), $y$ is " $i$ " or " $\mathrm{e}$ " if the specular reflection is included or excluded in the measurement (facultative), and $z$ denotes the observation geometry ("d" for an integrating sphere collecting light over the hemisphere, or an angle in degree for a detector capturing light in a specific direction).

Finally, independently of the selected geometries, the internal reflectance at the medium side, $r_{d}$, is the Lambertian reflectance of the interface since the medium is diffusing:

$$
r_{d}=r_{10}=1-\frac{1-r_{01}}{n^{2}}
$$

The transfer matrices representing the interfaces at the front and back sides, where the illumination-observation geometries are possibly different, are respectively

$$
\mathbf{F}=\frac{1}{t_{\text {in }}}\left(\begin{array}{cc}
1 & -r_{d} \\
r_{s} & t_{\text {in }} t_{\text {out }}-r_{s} r_{d}
\end{array}\right)
$$

and

$$
\mathbf{F}^{\prime}=\frac{1}{t_{\text {out }}^{\prime}}\left(\begin{array}{cc}
1 & -r_{s}^{\prime} \\
r_{d} & t_{\text {out }}^{\prime} t_{\text {in }}^{\prime}-r_{d} r_{s}^{\prime}
\end{array}\right)
$$

Knowing the intrinsic transfer matrix $\mathbf{M}$ representing the diffusing layer (without interfaces) the matrix representing the layer, with interfaces, is given by

Table 1. Geometry-Dependent Interface Parameters for Different Typical Geometries

\begin{tabular}{lccc}
\hline Measuring Geometry & $r_{s}$ & $t_{\text {in }}$ & $t_{\text {out }}$ \\
\hline d:d & $r_{01}$ & $t_{01}$ & $t_{10}$ \\
di: $8^{\circ}$ & $R_{10}\left(8^{\circ}\right)$ & $t_{01}$ & $T_{01}\left(8^{\circ}\right) / n^{2}$ \\
de: $8^{\circ}$ & 0 & $t_{01}$ & $T_{01}\left(8^{\circ}\right) / n^{2}$ \\
$8^{\circ}: \mathrm{di}$ & $R_{10}\left(8^{\circ}\right)$ & $T_{01}\left(8^{\circ}\right)$ & $t_{10}$ \\
$8^{\circ}: \mathrm{de}$ & 0 & $T_{01}\left(8^{\circ}\right)$ & $t_{10}$ \\
$45^{\circ}: 0^{\circ}$ & 0 & $T_{01}\left(45^{\circ}\right)$ & $T_{01}\left(0^{\circ}\right) / n^{2}$ \\
$0^{\circ}: 45^{\circ}$ & 0 & $T_{01}\left(0^{\circ}\right)$ & $T_{01}\left(45^{\circ}\right) / n^{2}$ \\
\hline
\end{tabular}

$$
\mathbf{P}=\mathbf{F M F}^{\prime}=\frac{1}{T}\left(\begin{array}{cc}
1 & -R^{\prime} \\
R & T T^{\prime}-R R^{\prime}
\end{array}\right) .
$$

The transfer factors $R, T, R^{\prime}$, and $T^{\prime}$ are obtained by computing this matrix product, then using formulas (7)-(10). Their analytical expansion yields the well-known formulas relating the reflectances and transmittances of the layer with and without interfaces []ㅜ:

$$
\begin{aligned}
R & =r_{s}+\frac{1}{\Delta} t_{\text {in }} t_{\text {out }}\left[\rho-r_{d}\left(\rho \rho^{\prime}-\tau \tau^{\prime}\right)\right], \\
T & =\frac{1}{\Delta} t_{\text {in }} \tau t_{\text {out }}^{\prime}, \\
R^{\prime} & =r_{s}^{\prime}+\frac{1}{\Delta} t_{\text {in }}^{\prime} t_{\text {out }}^{\prime}\left[\rho^{\prime}-r_{d}\left(\rho \rho^{\prime}-\tau \tau^{\prime}\right)\right], \\
T^{\prime} & =\frac{1}{\Delta} t_{\text {in }}^{\prime} \tau^{\prime} t_{\text {out }},
\end{aligned}
$$

with $\Delta=\left(1-r_{d} \rho\right)\left(1-r_{d} \rho^{\prime}\right)-r_{d}^{2} \tau \tau^{\prime}$.

In practice, these transfer factors are those that are measured, and we want to obtain the intrinsic transfer factors of the diffusing layer (without interfaces). We can use the following equation, issued from Eq. (17):

$$
\mathbf{M}=\mathbf{F}^{-1} \mathbf{P F}^{-1},
$$

and deduce the intrinsic transfer factors of the diffusing layer $\rho, \rho^{\prime}, \tau$, and $\tau^{\prime}$ using Eqs. (므)-(10).

\section{DUPLEX PRIMARY REFLECTANCE- TRANSMITTANCE MODEL}

The model developed in the previous section for a nonsymmetric diffusing layer with interfaces can be extended to layers with nonhomogeneous surfaces, typically paper substrate coated with halftone ink layers. For a paper coated on one side with a halftone containing $N$ primaries, we subdivide the paper area into $N$ subareas represented by different matrices, corresponding to the different primaries with respective surface coverage $a_{i}(i=1, \ldots, N)$. For a paper coated with halftones on its two sides, its area is subdivided into $N^{2}$ subareas, again represented by different matrices, corresponding to the different combinations of front and back primaries with respective surface coverage $a_{i} a_{j}(i=1, \ldots, N$, and $j=1, \ldots, N)$, called duplex primaries. Figure $\underline{3}$ shows an example of duplex halftone print with two primaries on each face, therefore four duplex primaries.

In the following, we will consider CMY halftones, containing eight Neugebauer primaries respectively labeled from 1 to 8: white (the areas with no ink), cyan, magenta, yellow, red (magenta and yellow), green (cyan and yellow), blue (cyan and magenta), and black (cyan, magenta and yellow). Denoting as $c, m$, and $y$ the surface coverages of cyan, magenta, and yellow inks, respectively, the surface coverages $a_{1}$ to $a_{8}$ of the eight primaries are deduced from Demichel's equations:

$$
\begin{array}{ll}
a_{1}=(1-c)(1-m)(1-y), & a_{5}=(1-c) m y, \\
a_{2}=c(1-m)(1-y), & a_{6}=c(1-m) y, \\
a_{3}=(1-c) m(1-y), & a_{7}=c m(1-y), \\
a_{4}=(1-c)(1-m) y, & a_{8}=c m y .
\end{array}
$$




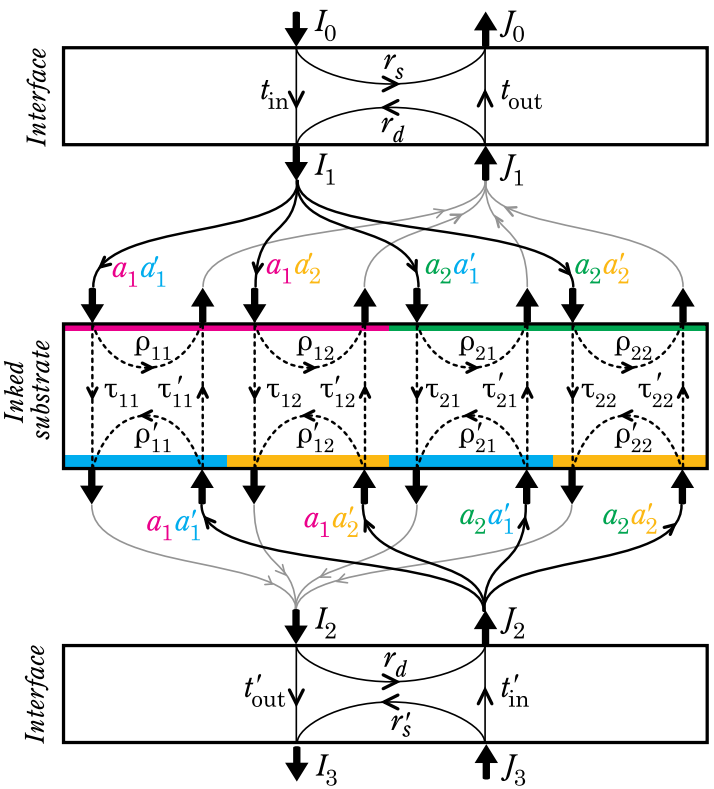

Fig. 3. Flux transfers between the front interface, the inked substrate containing four duplex primaries (combination of two primaries on each face), and the back interface.

With CMY halftones, we can have up to eight primaries on each side, therefore up to $8 \times 8=64$ duplex primaries.

In duplex halftone prints, the inked substrate is modeled as a juxtaposition of duplex primaries. The diffuse light is distributed between the different duplex primaries according to their respective surface coverage. Then, the flux propagates in each duplex primary independently of each other duplex primary, like in the Neugebauer model [34]. The transfer matrix $\mathbf{M}_{H}$ representing the inked substrate is written

$$
\mathbf{M}_{H}=\frac{1}{\tau_{H}}\left(\begin{array}{cc}
1 & -\rho_{H}^{\prime} \\
\rho_{H} & \tau_{H} \tau_{H}^{\prime}-\rho_{H} \rho_{H}^{\prime}
\end{array}\right)
$$

where, by using the generic letter $\omega$ in place of $\rho, \rho^{\prime}, \tau$, or $\tau^{\prime}$,

$$
\omega_{H}=\sum_{i, j} a_{i} a_{j}^{\prime} \omega_{i j}
$$

This can be easily shown by extending the graph of Fig. 3 to the 64 primaries and writing the two following equations, similar to Eqs. (1):

$$
\begin{aligned}
& J_{1}=\left(\sum_{i, j} a_{i} a_{j}^{\prime} \rho_{i j}\right) I_{1}+\left(\sum_{i j} a_{i} a_{j}^{\prime} \tau_{i j}^{\prime}\right) J_{2}, \\
& I_{2}=\left(\sum_{i, j} a_{i} a_{j}^{\prime} \tau_{i j}\right) I_{1}+\left(\sum_{i, j} a_{i} a_{j}^{\prime} \rho_{i j}^{\prime}\right) J_{2} .
\end{aligned}
$$

Moreover, by following step by step the line of reasoning from Eqs. (1) to (5), we can observe that Eq. (3) shows a matrix decomposition of the transfer matrix. Similar decomposition is possible with duplex halftones and enables separating the surface coverages from the transfer factors of the substrate and inks by defining the line vectors

$$
\mathbf{a}=\left(\begin{array}{lll}
a_{1} & \cdots & a_{8}
\end{array}\right), \mathbf{a}^{\prime}=\left(\begin{array}{lll}
a_{1}^{\prime} & \cdots & a_{8}^{\prime}
\end{array}\right), \mathbf{z}=\left(\begin{array}{lll}
0 & \cdots & 0
\end{array}\right) .
$$

and the $8 \times 8$ matrices

$$
\begin{aligned}
& \boldsymbol{\rho}=\left\{\rho_{i j}\right\}, \quad \boldsymbol{\tau}=\left\{\tau_{i j}\right\}, \\
& \boldsymbol{\rho}^{\prime}=\left\{\rho_{i j}^{\prime}\right\}, \quad \boldsymbol{\tau}^{\prime}=\left\{\tau_{i j}^{\prime}\right\} .
\end{aligned}
$$

We can write Eqs. (23) as

$$
\begin{aligned}
& J_{1}=\left(\mathbf{a} \rho \mathbf{a}^{\prime T}\right) I_{1}+\left(\mathbf{a} \tau^{\prime} \mathbf{a}^{\prime T}\right) J_{2}, \\
& I_{2}=\left(\mathbf{a} \tau \mathbf{a}^{\prime T}\right) I_{1}+\left(\mathbf{a} \rho^{\prime} \mathbf{a}^{\prime T}\right) J_{2},
\end{aligned}
$$

where superscript $T$ denotes the transpose operator. We obtain the following version of Eq. (3):

$$
\left(\begin{array}{l}
I_{1} \\
J_{1}
\end{array}\right)=\frac{1}{\mathbf{a} \tau \mathbf{a}^{\prime T}}\left(\begin{array}{cc}
0 & 1 \\
\mathbf{a} \tau \mathbf{a}^{\prime T} & \mathbf{a} \rho \mathbf{a}^{\prime T}
\end{array}\right)\left(\begin{array}{cc}
0 & \mathbf{a} \tau^{\prime} \mathbf{a}^{\prime T} \\
1 & -\mathbf{a} \rho^{\prime} \mathbf{a}^{\prime T}
\end{array}\right)\left(\begin{array}{c}
I_{2} \\
J_{2}
\end{array}\right),
$$

which can also be decomposed as

$$
\mathbf{M}_{H}=\frac{1}{\tau_{H}} \mathbf{A} \cdot\left(\begin{array}{cc}
\mathbf{0}_{8,8} & \mathbf{1}_{8,8} \\
\boldsymbol{\tau} & \boldsymbol{\rho}
\end{array}\right) \cdot \mathbf{A}^{\prime T} \mathbf{A} \cdot\left(\begin{array}{cc}
\mathbf{0}_{8,8} & \boldsymbol{\tau}^{\prime} \\
\mathbf{1}_{8,8} & -\boldsymbol{\rho}^{\prime}
\end{array}\right) \cdot \mathbf{A}^{\prime T},
$$

where $\mathbf{0}_{8,8}$ denotes the $8 \times 8$ zero matrix, $\mathbf{1}_{8,8}$ the $8 \times 8$ matrix of ones, and

$$
\mathbf{A}=\left(\begin{array}{cc}
\mathbf{a} & \mathbf{z} \\
\mathbf{z} & \mathbf{a}
\end{array}\right), \quad \mathbf{A}^{\prime}=\left(\begin{array}{cc}
\mathbf{a}^{\prime} & \mathbf{z} \\
\mathbf{z} & \mathbf{a}^{\prime}
\end{array}\right)
$$

Finally, the transfer matrix representing the duplex print, with its interfaces, is given by

$$
\mathbf{P}_{H}=\mathbf{F} \cdot \mathbf{M}_{H} \cdot \mathbf{F}^{\prime},
$$

and its transfer factors $R_{H}, R_{H}^{\prime}, T_{H}$, and $T_{H}^{\prime}$ are obtained using the formulas (7) to (10). Their analytical expansion yields similar formulas as (18) by replacing $\rho, \rho^{\prime}, \tau$, and $\tau^{\prime}$ (which represented the diffusing medium) with $\rho_{H}, \rho_{H}^{\prime}, \tau_{H}$, and $\tau_{H}^{\prime}$ (which now represent the inked paper):

$$
\begin{aligned}
R_{H} & =r_{s}+\frac{1}{\Delta} t_{\text {in }} t_{\text {out }}\left[\rho_{H}-r_{d}\left(\rho_{H} \rho_{H}^{\prime}-\tau_{H} \tau_{H}^{\prime}\right)\right], \\
T_{H} & =\frac{1}{\Delta} t_{\text {in }} t_{\text {out }}^{\prime} \tau_{H}, \\
R_{H}^{\prime} & =r_{s}^{\prime}+\frac{1}{\Delta} t_{\text {in }}^{\prime} t_{\text {out }}^{\prime}\left[\rho_{H}^{\prime}-r_{d}\left(\rho_{H} \rho_{H}^{\prime}-\tau_{H} \tau_{H}^{\prime}\right)\right], \\
T_{H}^{\prime} & =\frac{1}{\Delta} t_{\text {in }}^{\prime} t_{\text {out }} \tau_{H}^{\prime},
\end{aligned}
$$

with $\Delta=\left(1-r_{d} \rho_{H}\right)\left(1-r_{d} \rho_{H}^{\prime}\right)-r_{d}^{2} \tau_{H} \tau_{H}^{\prime}$.

These equations are not equivalent to those underlying the first reflectance and transmittance prediction model for duplex halftone prints presented in $[24,35]$, which was inspired of the Williams-Clapper model extended to halftones or of the Clapper-Yule model if one ignores the orientation of light in the ink layers. Indeed, there are several conceptual and practical differences between this model and the DPRT model, regarding optical properties of the inks and the way the optical dot gain is modeled. In order to clarify the comparison, we decided to review the Clapper-Yule-inspired model with the matrix approach in Section 7 , and to compare the two models in Section 8. 


\section{CALIBRATION OF THE DUPLEX PRIMARY REFLECTANCE- TRANSMITTANCE MODEL}

The DPRT model is made in two steps. The first step is the determination of the spectral transfer factors of each duplex primary. The second step is the ink spreading assessment (mechanical dot gain).

For obtaining the spectral transfer factors of the duplex primaries, the 64 duplex primaries $i j(i, j=1, \ldots, 8)$ are printed and their front and back reflectances $R_{i j}$ and $R_{i j}^{\prime}$, and backward and forward transmittances $T_{i j}$ and $T_{i j}^{\prime}$ are measured. This makes 256 measurements, from which are created 64 transfer matrices $\mathbf{P}_{i j}$. According to our model, each matrix can be decomposed as

$$
\mathbf{P}_{i j}=\mathbf{F M}_{i j} \mathbf{F}^{\prime}
$$

where $\mathbf{F}$ and $\mathbf{F}^{\prime}$ represent the front and back interfaces given by Eqs. (15) and (16), and $\mathbf{M}_{i j}$ denotes the intrinsic transfer matrix of the duplex primary (i.e., of the inked paper without interfaces). This latter is therefore given by

$$
\mathbf{M}_{i j}=\mathbf{F}^{-1} \mathbf{P}_{i j} \mathbf{F}^{-1},
$$

and the intrinsic transfer factors $\rho_{i j}, \rho_{i j}^{\prime}, \tau_{i j}$, and $\tau_{i j}^{\prime}$ are obtained using Eqs. (7)-(10).

Note that when the paper is symmetrical and the same inks are printed on both sides, we have $\rho_{i j}=\rho_{j i}^{\prime}$ and $\tau_{i j}=\tau_{j i}^{\prime}$. We thus observe that $R_{i j}=R_{j i}^{\prime}$ and $T_{i j}=T_{j i}^{\prime}$, which reduces the number of measurements to 128 instead of 256 .

For the ink spreading assessment, we follow the method proposed by Hersch et al. [36], also detailed in [37]. It relies on ink spreading functions giving the correspondence between nominal and effective surface coverages and taking into account the fact that an ink may spread differently on the paper or on top of another ink. The calibration of the ink spreading functions is based on single-face halftones.

First of all, a set of 36 single-ink halftones patches is printed on the front face of the paper. They correspond to the combinations of one ink $u$ at nominal surface coverages $a=0.25$, 0.5 or 0.75 , and one solid primary $v$ obtained by printing each other ink at a surface coverage 0 or 1 (this list of halftones is displayed in [37]).

Each of these halftones contains two primaries corresponding to the areas where primary $v$ is alone and areas where ink $u$ is superposed to primary $v$ (primary $u+v$, with effective surface coverage denoted as $x$ ). We can measure the spectral reflectance $R_{u / v}^{(m)}(\lambda)$ of this halftone, or its spectral transmittance $T_{u / v}^{(m)}(\lambda)$, or create the spectral transfer matrix $\mathbf{A}_{u / v}^{(m)}(\lambda)$ from its four measured transfer factors. The corresponding predicted quantities are respectively denoted $R_{u / v}^{(x)}(\lambda), T_{u / v}^{(x)}(\lambda)$, and $\mathbf{A}_{u / v}^{(m)}(\lambda)$. They are predicted from Eq. ( $\underline{30)}$ where, according to Eq. (22), the terms $\rho_{H}, \rho_{H}^{\prime}, \tau_{H}$, and $\tau_{H}^{\prime}$ are of the form

$$
\omega_{H}=(1-x) \omega_{v}+x \omega_{u+v} .
$$

The effective surface coverages are obtained by minimizing the quadratic difference between the measured and predicted spectral reflectances:

$$
\tilde{a}_{u / v}^{(R)}=\underset{0 \leq x \leq 1}{\arg \min } \sum_{\lambda \in \text { vis }}\left[R_{u / v}^{(m)}(\lambda)-R_{u / v}^{(x)}(\lambda)\right]^{2},
$$

where "vis" denotes the visible spectrum of light. They can also be obtained by minimizing the equivalent CIELAB $\Delta E_{94}$ color distance between them, obtained by converting them to CIE-XYZ tristimulus values calculated with a D65 illuminant for the $2^{\circ}$ standard observer, and then into CIELAB color coordinates using as white reference the spectral reflectance of the unprinted paper illuminated with the D65 illuminant [38]:

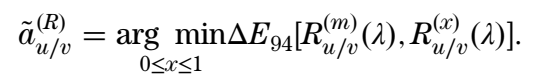

Equivalent minimization equations can be written from measured and predicted transmittances.

The matrix approach also allows direct minimization of the "distance" between the transfer matrices, $\mathbf{A}_{u / v}^{(m)}(\lambda)$ and $\mathbf{A}_{u / v}^{(x)}(\lambda)$, created from the measured spectral transfer factors, respectively from the predicted ones:

$$
\tilde{a}_{u / v}=\underset{0 \leq x \leq 1}{\arg \min }\left(\prod_{\lambda \in \text { vis }}\left\|\mathbf{A}_{u / v}^{(m)}(\lambda)-\mathbf{A}_{u / v}^{(x)}(\lambda)\right\|_{2}\right) .
$$

The distance between the two matrices is explained in Appendix $\underline{\mathrm{C}}$.

By selecting either minimization method, we obtain the 36 effective surface coverages associated with the 36 calibration halftones. For each set of ink $u$ and primary $v$, we have three effective surface coverages corresponding to the nominal coverages $0.25,0.5$, and 0.75 . By linear interpolation, we defined the ink spreading function $f_{u / v}(a)$ giving the effective coverage of ink $u$ on top of primary $v$ for any surface coverage $a$. We thus have 12 ink spreading functions.

For an application based on a symmetric paper (two identical faces), printed with the same inks on its two sides, there is no need to calibrate the ink spreading curves on the back side. Otherwise, the 36 calibration patches need to be printed on the back side, and the procedure described above needs to be repeated in order to obtain the 12 inks spreading functions $f_{u / v}^{\prime}(a)$

Once the ink spreading functions have been obtained, the spectral reflectance of any duplex halftone can be predicted. Let us consider a halftone on the front side where the cyan, magenta, and yellow ink are printed at the nominal surface coverages $c_{0}, m_{0}$, and $y_{0}$. These surface coverage numbers are converted into effective ink surface coverages $c, m$, and $y$ by a weighted average of the ink spreading functions in order to account for the superposition-dependent ink spreading, by performing a few iterations with the following equations:

$$
\begin{aligned}
c= & (1-m)(1-y) f_{c / w}\left(c_{0}\right)+m(1-y) f_{c / m}\left(c_{0}\right) \\
& +(1-m) y f_{c / y}\left(c_{0}\right)+m y f_{c / m+y}\left(c_{0}\right), \\
m= & (1-c)(1-y) f_{m / w}\left(m_{0}\right)+c(1-y) f_{m / c}\left(m_{0}\right) \\
& +(1-c) y f_{m / y}\left(m_{0}\right)+c y f_{m / c+y}\left(m_{0}\right), \\
y= & (1-c)(1-m) f_{y / w}\left(y_{0}\right)+c(1-m) f_{y / c}\left(y_{0}\right) \\
& +(1-c) m f_{y / m}\left(y_{0}\right)+c m f_{y / c+m}\left(y_{0}\right) .
\end{aligned}
$$

For the first iteration, $c=c_{0}, m=m_{0}$, and $y=y_{0}$ are the initial values on the right side of the equations. The obtained values of $c, m$, and $y$ are then inserted again into the right side of the equations, which gives new values of $c, m, y$, and so on, until the values of $c, m, y$ stabilize. They are then plugged into Eqs. (20) in order to obtain the effective surface coverages $a_{i}$ 
of the eight primaries on the front side. The same procedure is followed for the halftone on the back side by using, if necessary, the ink spreading functions $f_{u / v}^{\prime}(a)$ instead of the ink spreading functions $f_{u / v}(a)$. Once the effective surface coverages $\alpha_{j}^{\prime}$ of the eight primaries on the back side are computed, the spectral reflectances and transmittances of the duplex halftone print can be computed thanks to Eqs. (22) and (30).

\section{DUPLEX CLAPPER-YULE MODEL}

The first model proposed in the literature for predicting the reflectance and transmittance of duplex halftone prints $[24,35]$ was based on the assumption that the halftone ink layer is nonscattering and well distinct from the paper substrate, following the assumptions made by Williams and Clapper in their work on photographs [7], and by Clapper and Yule in their work on halftone prints [6]. In order to compare this model that we call the "DCY model" with the DPRT model introduced in this paper, we propose to review it by using transfer matrices.

Let us first consider a solid layer of a given primary. It has a transmittance $t_{k}$, and a reflectance zero since the inks are nonscattering. The inks are also assumed to have the same refractive index as the paper substrate: the ink-paper interface has therefore no optical effect and can be ignored. The ink layer and the ink-air interfaces are considered together as one component, the \{air-ink interface + ink $\}$ component, represented by the transfer matrix $\mathbf{F}_{k}$. This latter is obtained by multiplying the matrix $\mathbf{F}$ representing the interface, given by Eq. (15), and the matrix representing the ink layer:

$$
\mathbf{F}_{k}=\mathbf{F} \cdot\left(\begin{array}{cc}
1 / t_{k} & 0 \\
0 & t_{k}
\end{array}\right)=\frac{1}{t_{\text {in }} t_{k}}\left(\begin{array}{cc}
1 & -r_{d} t_{k}^{2} \\
r_{s} & \left(t_{\text {in }} t_{\text {out }}-r_{s} r_{d}\right) t_{k}^{2}
\end{array}\right) .
$$

The transfer matrix representing the print, i.e., the paper substrate bordered by \{air-ink interface + ink\} on the front side and by the paper-air interface at the back side, is given by

$$
\mathbf{P}_{k}=\mathbf{F}_{k} \mathbf{M} \mathbf{F}^{\prime}=\mathbf{F} \cdot\left(\begin{array}{cc}
1 / t_{k} & 0 \\
0 & t_{k}
\end{array}\right) \cdot \mathbf{M} \mathbf{F}^{\prime}
$$

The extension of this equation to CMY halftones with respective transmittances $t_{k}$ and coverages $a_{k}(k=1, \ldots, 8)$ is made as follows. At the front side, the incident flux $I_{0}$ splits into eight subfluxes $I_{0, k}$ flowing in parallel in the eight primary areas. Likewise, the incident flux $J_{1}$ at the back side splits into eight subfluxes $J_{1, k}$. Figure 4 illustrates the flux transfers within a duplex print where the halftones on the front and back faces contain two primaries.

By extending the graph of Fig. $\underline{4}$ to eight primaries on the front side, we could easily write the following two equations, similar to Eqs. (1) :

$$
\begin{aligned}
& J_{0}=r_{s} I_{0}+t_{\text {out }} \alpha J_{1}, \\
& I_{1}=t_{\text {in }} \alpha I_{0}+r_{d} \beta J_{1},
\end{aligned}
$$

with

$$
\alpha=\sum_{k=1}^{8} a_{k} t_{k}
$$

and

$$
\beta=\sum_{k=1}^{8} a_{k} t_{k}^{2}
$$

The transfer matrix representing the \{interface + halftone layer component at the front side is therefore similar to Eq. (5):

$$
\mathbf{F}_{H}=\frac{1}{t_{\text {in }} \alpha}\left(\begin{array}{cc}
1 & -r_{d} \beta \\
r_{s} & t_{\text {in }} t_{\text {out }} \alpha^{2}-r_{s} r_{d} \beta
\end{array}\right)
$$

As in the DPRT model, we can decompose this matrix as a product of rectangular matrices and separate the coverages from the transfer factors of the substrate and inks. By defining the following line vectors,

$$
\begin{aligned}
\mathbf{a} & =\left(\begin{array}{lll}
a_{1} & \cdots & a_{8}
\end{array}\right), \\
\mathbf{r} & =r_{s}\left(\begin{array}{lll}
1 & \cdots & 1
\end{array}\right) \\
\mathbf{t} & =t_{\text {in }}\left(\begin{array}{lll}
t_{1} & \cdots & t_{8}
\end{array}\right), \\
\mathbf{t}^{\prime} & =t_{\text {out }}\left(\begin{array}{lll}
t_{1} & \cdots & t_{8}
\end{array}\right), \\
\mathbf{r}^{\prime} & =r_{d}\left(\begin{array}{lll}
t_{1}^{2} & \cdots & t_{8}^{2}
\end{array}\right), \\
\mathbf{I}_{0} & =\left(\begin{array}{lll}
I_{0,1} & \cdots & I_{0,8}
\end{array}\right), \\
\mathbf{J}_{1} & =\left(\begin{array}{lll}
J_{1,1} & \cdots & J_{1,8}
\end{array}\right)
\end{aligned}
$$

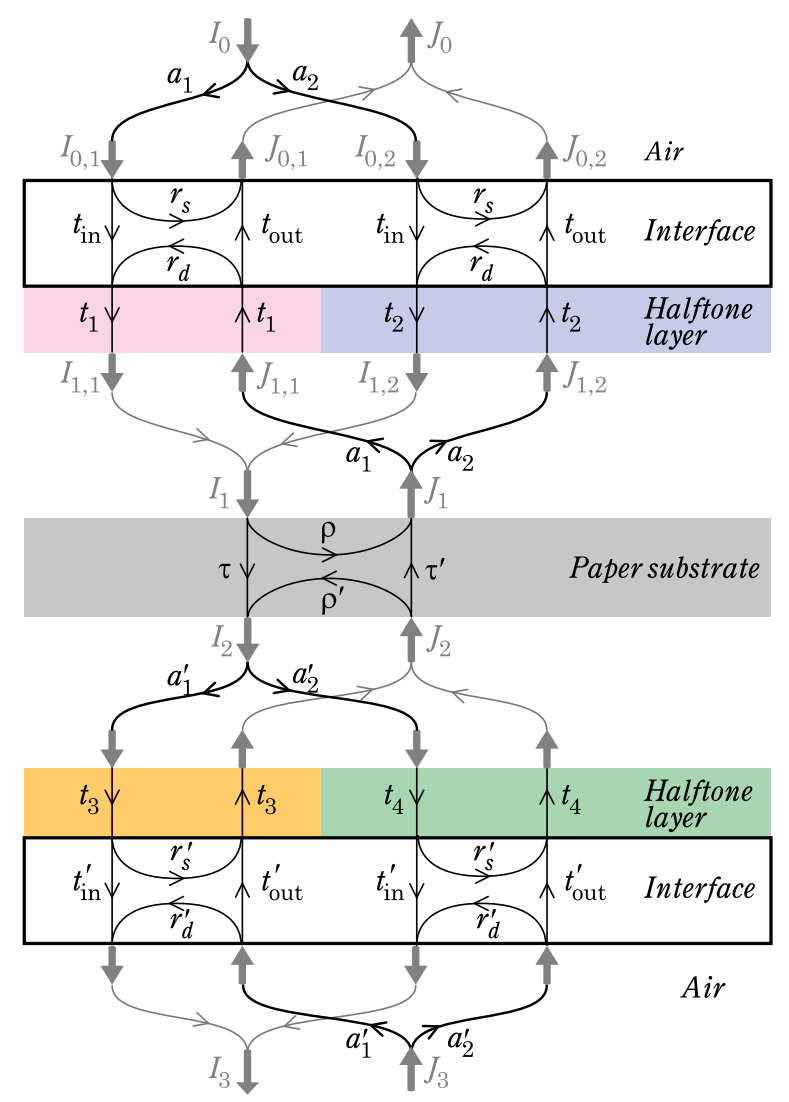

Fig. 4. Flux transfers between the \{interface + halftone layer component, the paper substrate and the \{halftone layer + interface $\}$ component in a duplex halftone print where each halftone contains two primaries. 
Eqs. (40) can be written

$$
\begin{aligned}
& J_{0}=\mathbf{r} \mathbf{I}_{0}^{T}+\mathbf{t}^{\prime} \mathbf{J}_{1}^{T}=\mathbf{r a}^{T} I_{0}+\mathbf{t}^{\prime} \mathbf{a}^{T} J_{1}, \\
& I_{1}=\mathbf{t} \mathbf{I}_{0}^{T}+\mathbf{r}^{\prime} \mathbf{J}_{1}^{T}=\mathbf{t a}^{T} I_{0}+\mathbf{r}^{\prime} \mathbf{a}^{T} J_{1} .
\end{aligned}
$$

Then, by noticing that $\mathbf{r a}^{T}=\mathbf{a r}^{T}$ and $\mathbf{t a}^{T}=\mathbf{a t}^{T}=t_{\text {in }} \alpha$, we obtain the following version of Eq. (3):

$$
\left(\begin{array}{l}
I_{0} \\
J_{0}
\end{array}\right)=\frac{1}{t_{\mathrm{in}} \alpha}\left(\begin{array}{cc}
0 & 1 \\
\mathbf{a t}^{T} & \mathbf{a r}^{T}
\end{array}\right)\left(\begin{array}{cc}
0 & \mathbf{t}^{\prime} \mathbf{a}^{T} \\
1 & -\mathbf{r}^{\prime} \mathbf{a}^{T}
\end{array}\right)\left(\begin{array}{l}
I_{1} \\
J_{1}
\end{array}\right)
$$

The transfer matrix $\mathbf{F}_{H}$ is therefore given by the following product of $2 \times 9$ and $9 \times 2$ matrices:

$$
\mathbf{F}_{H}=\frac{1}{t_{\text {in }} \alpha} \mathbf{A}\left(\begin{array}{cc}
0 & 1 \\
\mathbf{t}^{T} & \mathbf{r}^{T}
\end{array}\right)\left(\begin{array}{cc}
0 & \mathbf{t}^{\prime} \\
1 & -\mathbf{r}^{\prime}
\end{array}\right) \mathbf{A}^{T}
$$

where

$$
\mathbf{A}=\left(\begin{array}{cc}
1 & \mathbf{0}_{1,8} \\
0 & \mathbf{a}
\end{array}\right)
$$

For the halftone ink layer printed on the back face of the paper (with the primary coverages $a_{k}^{\prime}$ ), the transfer matrix representing the \{halftone layer + interface $\}$ component is

$$
\mathbf{F}_{H}^{\prime}=\frac{1}{t_{\text {out }} \alpha^{\prime}}\left(\begin{array}{cc}
1 & -r_{s} \\
r_{d} \beta^{\prime} & t_{\text {in }} t_{\text {out }} \alpha^{\prime 2}-r_{s} r_{d} \beta^{\prime}
\end{array}\right),
$$

where $\alpha^{\prime}$ and $\beta^{\prime}$ are defined as $\alpha$ and $\beta$ in terms of the coverages $a_{k}^{\prime}$.

Finally, the transfer matrix representing the duplex print, $\mathbf{P}_{H}$, is the product of the transfer matrices $\mathbf{F}_{H}, \mathbf{M}$, and $\mathbf{F}_{H}^{\prime}$ respectively given by Eqs. (41), (12), and (6)

$$
\mathbf{P}_{H}=\mathbf{F}_{H} \cdot \mathbf{M} \cdot \mathbf{F}_{H}^{\prime}
$$

The analytical calculation of $\mathbf{P}_{H}$, then the use of formulas (7)-(10), yields the following analytical reflectance and transmittance expressions for the duplex print:

$$
\begin{aligned}
& R_{H}=r_{s}+\frac{1}{\Delta} \alpha^{2} t_{\text {in }} t_{\text {out }}\left[\rho-\beta r_{d}\left(\rho \rho^{\prime}-\tau \tau^{\prime}\right)\right], \\
& T_{H}=\frac{1}{\Delta} \alpha \alpha^{\prime} t_{\text {in }} t_{\text {out }}^{\prime} \tau, \\
& R_{H}^{\prime}=r_{s}^{\prime}+\frac{1}{\Delta} \alpha^{\prime 2} t_{\text {in }}^{\prime} t_{\text {out }}^{\prime}\left[\rho^{\prime}-\beta^{\prime} r_{d}\left(\rho \rho^{\prime}-\tau \tau^{\prime}\right)\right], \\
& T_{H}^{\prime}=\frac{1}{\Delta} \alpha \alpha^{\prime} t_{\text {in }}^{\prime} t_{\text {out }} \tau^{\prime},
\end{aligned}
$$

with $\Delta=\left(1-\rho \beta r_{d}\right)\left(1-\rho^{\prime} \beta^{\prime} r_{d}\right)-\beta \beta^{\prime} r_{d}^{2} \tau \tau^{\prime}$.

The original Clapper-Yule model $[\underline{6}, \underline{39}]$ is a special case of this model, since it considers only the reflectance of a singleink halftone printed on the recto face. The halftone contains two primaries: ink and unprinted paper, with respective transmittance $t$ and 1 , and respective coverages $a$ and $1-a$. The substrate is considered as a background with reflectance layer $\rho_{g}$, which would correspond to the \{substrate layer + back interface] component in our model [40]:

$$
\rho_{g}=\rho+\frac{\tau^{2} r_{d}}{1-\rho r_{d}}
$$

The Clapper-Yule reflectance formula can be derived from the analytical expression of $R_{H}$ with $\alpha=1-a+a t$, $\beta=1-a+a t^{2}$, and $\rho_{g}$ given by Eq. (노):

$$
R_{\mathrm{CY}}=r_{s}+\frac{t_{\text {in }} t_{\text {out }} \rho_{g}(1-a+a t)^{2}}{1-r_{d} \rho_{g}\left(1-a+a t^{2}\right)} .
$$

The calibration of the DCY model needs to determine the spectral transfer factors of the substrate, the spectral transmittance of the eight primaries and their effective surface coverages.

As explained in Section 6, the intrinsic transfer matrix $\mathbf{M}$ representing the paper substrate is obtained by measuring the transfer factors of the paper sheet and using Eq. (19). Then, the transmittance of each primary is obtained as follows: a solid layer of the primary is printed on the paper, its transfer factors $R_{k}(\lambda), R_{k}^{\prime}(\lambda), T_{k}(\lambda)$, and $T_{k}^{\prime}(\lambda)$ are measured using a spectrophotometer, and the transfer matrix is created for each wavelength:

$$
\mathbf{P}_{k}=\frac{1}{T_{k}}\left(\begin{array}{cc}
1 & -R_{k}^{\prime} \\
R_{k} & T_{k} T_{k}^{\prime}-R_{k} R_{k}^{\prime}
\end{array}\right)
$$

According to the matrix decomposition of $\mathbf{P}_{k}$ given by Eq. (39), we have, in theory,

$$
\left(\begin{array}{cc}
1 / t_{k} & 0 \\
0 & t_{k}
\end{array}\right)=\mathbf{F}^{-1} \mathbf{P}_{k} \mathbf{F}^{-1} \mathbf{M}^{-1}
$$

and we can deduce $t_{k}$ from the diagonal matrix. Notice that it is possible, and frequently observed experimentally, that the numerical matrices at the right-hand of Eq. (52) do not yield a diagonal matrix, which means that the assumption of nonscattering ink is not strictly satisfied. This will be discussed in the next section.

Regarding the effective surface coverages in halftones, they are computed according to the method explained in Section 6 for the DPRT model. The effective surface coverages of the $3 \overline{6}$ calibration halftones are obtained by minimizing the difference between the predicted and measured spectra, i.e., the sum of squared differences between the spectra [see Eq. (34)] or the equivalent $\Delta E_{94}$ color distance [see Eq. (35)]. Note that the minimization equation (C4) is not allowed with the DCY: since we must consider different surface coverages of primaries in the reflectance and transmittance expressions for the calibration patches, we cannot create from them a transfer matrix being a function of single surface coverage.

\section{THEORETICAL AND EXPERIMENTAL COMPARISON OF THE MODELS}

The DPRT model and the DCY model are both based on transfer matrices but have several differences regarding the assumptions on the inks, the way the optical dot gain is modeled, and the number of measurements needed for calibration.

\section{A. Assumptions Regarding the Inks in the Duplex Clapper-Yule Model}

Equation (52) derives from the assumption that the inks are assumed to be nonscattering, i.e., to have a reflectance zero. 
In practice, however, we often observe that the obtained matrix is not a diagonal matrix, and that the top-left entry is not the inverse of the bottom-right entry. By way of illustration, we obtain the following matrix for a solid layer of cyan ink printed in inkjet on calendered nonfluorescing paper at $600 \mathrm{~nm}$ :

$$
\left(\begin{array}{cc}
1.9764 & -0.3193 \\
0.1469 & 0.5261
\end{array}\right)
$$

This clearly indicates that the assumptions underlying the Clapper-Yule model that the inks are not reflective is not verified. Alternatively, we could represent the primary layer by a classical transfer matrix (defined in terms of intrinsic reflectance $r_{k}$ and transmittance $t_{k}$, by assuming a symmetric layer) in place of the diagonal matrix in Eq. (39), i.e.,

$$
\mathbf{P}_{k}=\mathbf{F} \cdot \frac{1}{t_{k}}\left(\begin{array}{cc}
1 & -r_{k} \\
r_{k} & t_{k}^{2}-r_{k}^{2}
\end{array}\right) \cdot \mathbf{M F}^{\prime}
$$

The right-hand of Eq. (52) would therefore give a nondiagonal matrix, from which the $r_{k}$ and $t_{k}$ values could be deduced. However, our experience in inkjet printing on paper shows that this method is numerically instable, i.e., the values for $r_{k}$ and $t_{k}$ deduced from numerical matrices like Eq. (53) are aberrant: they often overpass 1 and may even be negative. Hence, we prefer considering the classical approach where the ink layer has a zero reflectance. We can either use reflectance measurements or transmittance measurements to deduce the primary transmittances $t_{k}$. The expressions for the measured transfer factors are given by Eq. (48) by setting $\alpha=$ $t_{k}, \beta=t_{k}^{2}, \alpha^{\prime}=1$ and $\beta^{\prime}=1$.

If we want to predict the reflectance of a duplex halftone, we strongly recommend computing the primary transmittances from measurements of the front-side reflectance $R_{k}$, by using the following formula derived from Eq. (48):

$$
t_{k, R}=\sqrt{\frac{\left(R_{k}-r_{s}\right)\left(1-\rho^{\prime} r_{d}\right)}{U\left[t_{\mathrm{in}} t_{\mathrm{out}}+\left(R_{k}-r_{s}\right) r_{d}\right]}}
$$

with

$$
U=\rho-r_{d}\left(\rho \rho^{\prime}-\tau \tau^{\prime}\right)
$$

If we rather want to predict the transmittance of the duplex print, the following formula based on the measurement of a backward transmittance $T_{k}^{\prime}$ is preferable:

$$
t_{k, T}=\frac{\sqrt{\left(t_{\mathrm{in}}^{\prime} t_{\mathrm{out}} \tau\right)^{2}+4 T_{k}^{\prime 2}\left(1-\rho^{\prime} r_{d}\right) r_{d} U}-t_{\mathrm{in}}^{\prime} t_{\mathrm{out}} \tau}{2 T_{k}^{\prime} r_{d} U} .
$$

The spectral transmittances of primaries obtained from Eqs. (55) and (ㅎ6) may differ significantly, as shown in Fig. $\underline{5}$ for the seven colored primaries printed with the Canon PixmaPro 9500 inkjet printer on supercalendered paper. The most noticeable difference concerns all primaries containing yellow ink (i.e., yellow, red, green, and black). We might expect poor prediction accuracy when the transmittances $t_{k, R}$ given by Eq. (55) are used to predict the transmittance of prints, or conversely when the transmittances $t_{k, T}$ given by Eq. (56) are used to predict their reflectance. This was already

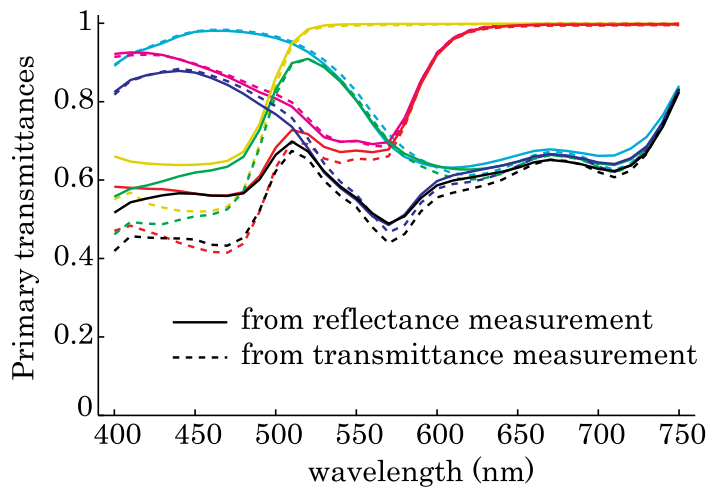

Fig. 5. Spectral transmittances of the cyan, magenta, yellow, red, green, blue, and black primaries deduced from reflectance (solid lines) or transmittance (dashed lines) measurements.

noticed in [35] and is clearly confirmed by our experiments based on the DCY model presented below. This can be an issue in applications where both reflectance and transmittance need to be considered, in particular when halftone prints are stacked: when describing the flux transfers between the different prints in the stacked, we need to consider different transmittances and surface coverages for the primaries of a same halftone according to whether the light is reflected or transmitted (this was the case, for example, in the work presented in [41] addressing stacks of films and papers).

All considerations above concern only the DCY model. The DPRT model makes no assumption regarding the reflectance and transmittance of the ink layers themselves.

\section{B. Optical Dot Gain}

Conceptually, the two models implicitly assume that light issued from one primary can reach, after reflection by the interface (in the DPRT model) or by the paper substrate (in the DCY model), any of the eight primary independently of the primaries met before. It means that the lateral propagation distance of light between two primaries is assumed much larger than the halftone period and theoretically restricts the use of the model to high-frequency halftones [39]. This was experimentally confirmed in [37], even though predictive performance remains acceptable for middle halftone frequencies (around 75 lpi in periodical cluster dot halftoning). Regarding the optical dot gain, we can conclude that the two models are nearly equivalent.

\section{Needed Measurements for Calibration}

As an advantage, the DCY model is much easier to calibrate than the DPRT model. With CMY halftones, it needs only eight measurements to get the primary transmittances on the front side, as well as eight other measurements on the back side if the paper is nonsymmetric (the primary transmittances may be different in this case). In contrast, the DPRT model needs in theory to measure the four flux transfers (front-side and back-side reflectances, forward and backward transmittances) of the 64 duplex primaries, which makes 256 measurements. However, the number of measurements may be strongly decreased by observing that, in practice, the forward and backward transmittances are equal, and that duplex primaries $i j$ and $j i$ have the same transmittance. Moreover, if the paper is symmetric, the front reflectance of the duplex primaries $i j$ equals the back reflectance of the primary $j i$. Finally, 
when the paper is opaque enough, the front reflectance of the duplex primaries $i j$ depends only on the primary $i$ printed at the front side (the transmittance still depends on both primaries $i$ and $j$ ). The calibration of the ink spreading curves is similar in the two models: it needs 36 reflectance measurements and 36 transmittance measurements.

\section{Experimental Verification of the Models}

In order to compare the prediction accuracy of the two models, we printed duplex halftones with the Canon Pro9500 inkjet printer on symmetrical, supercalendered, nonfluorescent paper APCO II from Scheufelen Company, Germany. Classical rotated cluster halftoning at 120 lpi was used for the two sides. The CIELAB $\Delta E_{94}$ color distance between measured and predicted spectra is used to assess the prediction quality. As the final target of these spectral prediction models is to predict colors, this metric is more relevant than the spectral difference to estimate a perceptible difference between predicted and measured spectra. It also gives an interpretable scale for accuracy assessment: we will consider that the prediction is good when the average $\Delta E_{94}$ value is lower than 1 .

On the front side, we printed eight bands of different colors whose coverages for the cyan, magenta, and yellow inks were randomly selected. On the back side, we printed the same eight bands rotated by $90^{\circ}$, thus forming a $8 \times 8$ grid of duplex patches. Each patch was measured in the reflectance mode and in the transmittance mode with the X-rite Color i7 spectrophotometer. The front-side and back-side reflectance measurements were based on the $\mathrm{d}: 8^{\circ}$ geometry, with no background beside the print. The forward and backward transmittance measurements were based on the $\mathrm{d}: 0^{\circ}$ geometry. The paper that we used is rather opaque, since its average transmittance is around $10 \%$ in the visible range. The ink on the back side of the paper is therefore almost invisible in the reflectance mode: if we compare the reflectance spectra of the paper with the different halftones on the back side using the $\Delta E_{94}$ metric, we obtain 0.25 units in average.

Due to the symmetry of the paper and the fact the same colors were printed on the front and back sides of the paper, there was no difference between the set of measured frontside reflectances and the set of measured back-side reflectances, nor between the sets of forward transmittances and of backward transmittances. Hence, only the front-side reflectances and the backward transmittances will be analyzed.

The calibration of the DPRT model was performed as described in Section $\underline{6}$ by using no ink spreading function, and by using ink spreading functions obtained through the minimization equations (34), (35), or (36). Equations (34) and (35) may be applied in the reflectance mode by considering the predictive formula of reflectance and reflectance measurements; these equations may also be applied in the transmittance mode. The DCY model was calibrated by computing the intrinsic transfer matrix of the paper substrate using Eq. (19), then by computing the ink transmittances according to Eqs. (55) and (56), from reflectance, respectively transmittance measurements. Once again, we first used no ink spreading function, then ink spreading functions obtained through the minimization equations (34) or (35), computed in the reflectance mode or the transmittance mode. Recall that the minimization equation (36) cannot be used with the DCY. We also tested each model by considering the nominal surface coverages of primaries (no ink spreading functions).

Table 2 presents the average $\Delta E_{94}$ values obtained for 64 duplex halftones with the DPRT and DCY models by using the different calibration options. Recall that only the effective coverages necessary to compute the ink spreading functions are fitted. No other free parameter is fitted. Without ink spreading functions, the two models are more accurate in the transmittance mode than the reflectance mode. With ink spreading functions, their prediction accuracy is comparable in both modes. It therefore seems that the effect of the ink spreading functions is stronger in the reflectance mode than in the transmittance mode.

With the DPRT model, good prediction accuracy is achieved with the three minimization Eqs. (34)-(36), the best accuracy being achieved with the equation based on the $\Delta E_{94}$ value, Eq. (35), which is therefore recommended. However, if we want to predict both reflectances and transmittances of duplex primaries by using single calibration, one can use the minimization equation (36) which yields almost as accurate predictions as those yielded by Eq. (34)

With the DCY model, we have similar accuracy as the DPRT model provided that reflectance predictions rely on parameters calibrated from reflectance measurements, or that transmittance predictions rely on parameters calibrated from transmittance measurements; otherwise, the prediction accuracy may be sensibly decreased. For instance, regarding the front-side reflectances predicted with the DCY model, we obtain an average $\Delta E_{94}$ value of 1.61 units with a calibration based on transmittance measurements (not presented in Table 1) instead of the 0.72 units obtained with a calibration based on reflectance measurements.

Finally, regarding the prediction of transmittance, despite the fact that the DPRT model looks sensibly more accurate in average than the DCY model (calibrations based on transmittance measurements), it is less accurate for the few patches for which accuracy is the poorest: the 95-percentile is around 2 units with the DPRT model and less than 1.5 with

Table 2. Spectral and Color Differences on 64 Duplex Patches ${ }^{a}$

\begin{tabular}{|c|c|c|c|c|}
\hline \multirow[b]{2}{*}{ Minimization Equation } & \multicolumn{2}{|c|}{ DPRT Model } & \multicolumn{2}{|c|}{ DCY Model } \\
\hline & Reflectance & Transmittance & Reflectance & Transmittance \\
\hline (none) & $1.33(2.00), 0.013^{b}$ & $1.12(1.69), 0.003^{c}$ & $1.67(2.57), 0.021^{b}$ & $1.14(1.63), 0.003^{c}$ \\
\hline$(34)$ & $0.79(1.47), 0.010^{b}$ & $0.86(2.04), 0.002^{c}$ & $0.72(1.39), 0.008^{b}$ & $1.01(1.47), 0.001^{c}$ \\
\hline$(35)$ & $0.66(1.21), 0.008^{b}$ & $0.77(2.00), 0.002^{c}$ & $0.73(1.38), 0.006^{b}$ & $0.85(1.24), 0.001^{c}$ \\
\hline (36) & $0.78(1.50), 0.00 \gamma^{d}$ & $0.93(2.04), 0.002^{d}$ & - & - \\
\hline
\end{tabular}

${ }^{a}$ The three numbers correspond respectively to: average $\Delta E_{94}$ value (95th percentile), $r m s$ spectral difference. The ink spreading functions are computed:

${ }^{b}$ from reflectance measurements,

${ }^{c}$ from transmittance measurements,

${ }^{d}$ from both reflectance and transmittance measurements (matrix minimization equation). 
the DCY model. The DCY model should therefore be preferred for transmittance predictions if the tolerance on the prediction accuracy is very low.

\section{CONCLUSIONS}

The DPRT model introduced in this paper is an original approach allowing the prediction of the reflectance and transmittance of duplex halftone prints with good accuracy, from a fully numerical matrix calibration. The transfer matrix formalism underlying this model was decisive for its derivation; it also highlights meaningfully the limitations of the classical Clapper-Yule approach, which assumes that the ink layers cannot reflect light by themselves: instead of obtaining a diagonal matrix as the model would expect, the nondiagonal matrices that we often obtain show that this assumption is not satisfied, either because the inks scatter light by themselves like toner in electrophotography printing, or because they penetrate the paper substrate and form with it a scattering colored layer. Compared to the Clapper-Yuleinspired model previously proposed in the literature, the duplex primary model needs more measurements for its calibration but, as an advantage, it enables accurate reflectance and transmittance predictions with a same set of parameter values while the Clapper-Yule-inspired model needs two distinct sets of parameter values for reflectance, respectively transmittance predictions. This is an appreciable advantage in every application where both reflectance and transmittance of a same halftone print are needed, especially to model the flux transfers between this print and other optical components on top of it: protecting glass plate, overlay, moiré revelator film. Our experiments based on inkjet prints shows that the double-mode calibration is slightly less accurate than the reflectance-only calibration mode for reflectance predictions, or the transmittance-only calibration mode for transmittance predictions, but it remains satisfactory since the average $\Delta E_{94}$ value computed between the predicted and measured spectral reflectances and transmittances is below 1 , which is typically considered as a good performance in the color reproduction domain

As an application of duplex prints, we can imagine innovative billboards or signages displaying different images according to whether they are illuminated from outside during the day or from inside at night. For such application, a design software including a calibrated predictive model is needed for calculating the halftones to print on the two faces in order to display the good colors in the two configurations.

\section{APPENDIX A: STACKS OF IDENTICAL NONSYMMETRIC COMPONENTS}

When symmetric or nonsymmetric components (e.g., paint layers, films, paper sheets, printed supports), all identical, are stacked with each other, we can obtain analytical expressions for the reflectance and transmittance of the stack as a function of the number of components, as shown in [11] through the example of printed films. Here, with slightly different equations, we go further by showing that the number of components may also be a real number.

Let us consider components represented by the same transfer matrix $\mathbf{M}$ defined as in Eq. (5) in terms of the transfer factors $r, r^{\prime}, t$, and $t^{\prime}$. The matrix representing a stack of $x$ components is $\mathbf{M}^{x}$. The two eigenvalues of $\mathbf{M}$ are

$$
\begin{aligned}
& \mu=\frac{1}{t}\left[1-\sqrt{r r^{\prime}}(\alpha+\beta)\right], \\
& \nu=\frac{1}{t}\left[1-\sqrt{r r^{\prime}}(\alpha-\beta)\right],
\end{aligned}
$$

with

$$
\alpha=\frac{1+r r^{\prime}-t t^{\prime}}{2 \sqrt{r r^{\prime}}}
$$

and

$$
\beta=\sqrt{\alpha^{2}-1}
$$

The associated eigenvectors can be respectively written $\left(r^{\prime}, 1-\mu t\right)^{T}$ and $\left(r^{\prime}, 1-\nu t\right)^{T}$. Therefore, $\mathbf{M}$ is decomposed as

$$
\begin{aligned}
\mathbf{M}= & \frac{1}{r^{\prime} t(\nu-\mu)}\left(\begin{array}{cc}
r^{\prime} & r^{\prime} \\
1-\mu t & 1-\nu t
\end{array}\right) \cdot\left(\begin{array}{ll}
\mu & 0 \\
0 & \nu
\end{array}\right) \\
& \cdot\left(\begin{array}{cc}
1-\nu t & -r^{\prime} \\
-(1-\mu t) & r^{\prime}
\end{array}\right),
\end{aligned}
$$

and $\mathbf{M}^{x}$ is obtained by raising $\mu$ and $\nu$ in the diagonal matrix to the power $x$. After expansion, one has

$$
\mathbf{M}^{x}=\frac{1}{2 \beta}\left(\begin{array}{cc}
\nu^{x}(\alpha+\beta)-\mu^{x}(\alpha-\beta) & -\left(\nu^{x}-\mu^{x}\right) \sqrt{r^{\prime} / r} \\
\left(\nu^{x}-\mu^{x}\right) \sqrt{r^{\prime} / r} & \mu^{x}(\alpha+\beta)-\nu^{x}(\alpha-\beta)
\end{array}\right) .
$$

By using formulas (7) to (10), we obtain the following transfer factor expressions:

$$
\begin{aligned}
R_{x} & =\frac{\left(\nu^{x}-\mu^{x}\right) \sqrt{r / r^{\prime}}}{\nu^{x}(\alpha+\beta)-\mu^{x}(\alpha-\beta)}, \\
T_{x} & =\frac{2 \beta}{\nu^{x}(\alpha+\beta)-\mu^{x}(\alpha-\beta)}, \\
R_{x}^{\prime} & =R_{x}\left(r^{\prime} / r\right), \\
T_{x}^{\prime} & =T_{x}\left(t^{\prime} / t\right)^{x},
\end{aligned}
$$

where $\mu, \nu, \alpha$, and $\beta$, given by Eqs. (A1) to (A3), depend on $r, r^{\prime}, t$, and $t^{\prime}$. Notice the following remarkable relation, valid for any $x$ value:

$$
\frac{1+R_{x} R_{x}^{\prime}-T_{x} T_{x}^{\prime}}{2 \sqrt{R_{x} R_{x}^{\prime}}}=\alpha
$$

As $x$ increases to infinity, the reflectance $R_{x}$ tends to the limit reflectance:

$$
R_{\infty}=\sqrt{\frac{r}{r^{\prime}}} \frac{1}{\alpha+\beta}=\sqrt{\frac{r}{r^{\prime}}}(\alpha-\beta) .
$$

Mathematically, there is no issue to consider that $x$ is real number. This makes sense with a diffusing medium: if $\mathbf{M}$ represents a layer with unit thickness, $\mathbf{M}^{x}$ represents a layer with thickness $x$. With nonscattering films (and collimated light [11]), the number of layers is necessarily an integer number. If $\mathbf{M}$ represents one film, $\mathbf{M}^{m}$ represents the stack of $m$ films, which can be considered as an effective homogeneous medium with thickness $m$, and $\mathbf{M}^{x}$ gives the upward and downward fluxes at any altitude $x$ in this effective layer. 


\section{APPENDIX B: KUBELKA-MUNK MODEL GENERALIZED TO NONSYMMETRIC MEDIA}

The formulas derived in Eqs. (A6) expressing the transfer factors of stacks of identical components enable retrieving the Kubelka-Munk hyperbolic reflectance and transmittance formulas, by considering that a homogenous layer of thickness $h$ is a stack of $N$ sublayers of thickness $h / N$ as $N$ tends to infinity. This was shown in [10] in the case of a symmetrical layer, by considering that every sublayer is symmetrical, and has a reflectance $r=h S / N$ and a transmittance $t=1-h(K+$ $S) / N$ where $K$ and $S$ are the absorption and backscattering coefficients of the medium. Here, we propose to review this line of reasoning by considering that the sublayers are still identical, but nonsymmetric by considering different scattering and absorption coefficients according to the direction of light: $S$ and $K$ when the light is oriented downward, and $S^{\prime}$ and $K^{\prime}$ when it is oriented upward. Each sublayer is therefore represented by the matrix

$$
\mathbf{M}_{h / N}=\frac{1}{t}\left(\begin{array}{cc}
1 & -r^{\prime} \\
r & t t^{\prime}-r r^{\prime}
\end{array}\right),
$$

with

$$
\begin{aligned}
r & =S \frac{h}{N}, \quad t=1-(K+S) \frac{h}{N}, \\
r^{\prime} & =S^{\prime} \frac{h}{N}, \quad t^{\prime}=1-\left(K^{\prime}+S^{\prime}\right) \frac{h}{N} .
\end{aligned}
$$

The transfer factors of the $N$ sublayers, therefore is the whole layer, are given by Eq. (A6) where $\alpha, \beta, \mu$, and $\nu$ become

$$
\begin{aligned}
\alpha_{h / N} & =\frac{K+K^{\prime}+S+S^{\prime}}{2 \sqrt{S S^{\prime}}}-\frac{h}{N} \frac{\left(K K^{\prime}+K S^{\prime}+K^{\prime} S\right)}{2 \sqrt{S S^{\prime}}}, \\
\beta_{h / N} & =\sqrt{\alpha_{h / N}^{2}-1}, \\
\mu_{h / N} & =\frac{1-\frac{h}{N} \sqrt{S S^{\prime}}\left(\alpha_{h / N}+\beta_{h / N}\right)}{1-(K+S) \frac{h}{N}}, \\
\nu_{h / N} & =\frac{1-\frac{h}{N} \sqrt{S S^{\prime}}\left(\alpha_{h / N}-\beta_{h / N}\right)}{1-(K+S) \frac{h}{N}} .
\end{aligned}
$$

As $N$ tends to infinity, using a classical result for the exponential function [42],

$$
\lim _{n \rightarrow+\infty}\left(1-\frac{x_{n}}{n}\right)^{n}=e^{-x}
$$

with

$$
x=\lim _{n \rightarrow+\infty} x_{n}
$$

and computing the limits

$$
\begin{aligned}
a & =\lim _{N \rightarrow+\infty} \alpha_{h / N}=\frac{K+K^{\prime}+S+S^{\prime}}{2 \sqrt{S S^{\prime}}}, \\
b & =\sqrt{a^{2}-1}, \\
\mu & =\lim _{N \rightarrow+\infty} \mu_{h / N}^{N}=\frac{e^{-\sqrt{S S^{\prime}}(a+b) h}}{e^{-(K+S) h}}, \\
\nu & =\lim _{N \rightarrow+\infty} \nu_{h / N}^{N}=\frac{e^{-\sqrt{S S^{\prime}}(a-b) h}}{e^{-(K+S) h},}
\end{aligned}
$$

one obtains the following expressions for the transfer factors of the whole layer, which are a generalization of the hyperbolic Kubelka-Munk formulas:

$$
\begin{aligned}
R & =\frac{\sqrt{S / S^{\prime}}}{a+b \operatorname{coth}\left(b \sqrt{S S^{\prime}} h\right)}, \\
T & =\frac{b e^{-(K+S) h / 2} e^{\left(K^{\prime}+S^{\prime}\right) h / 2}}{a \sinh \left(b \sqrt{S S^{\prime}} h\right)+b \cosh \left(b \sqrt{S S^{\prime}} h\right)}, \\
R^{\prime} & =\frac{\sqrt{S^{\prime} / S}}{a+b \operatorname{coth}\left(b \sqrt{S S^{\prime}} h\right)}, \\
T^{\prime} & =\frac{b e^{(K+S) h / 2} e^{-\left(K^{\prime}+S^{\prime}\right) h / 2}}{a \sinh \left(b \sqrt{S S^{\prime}} h\right)+b \cosh \left(b \sqrt{S S^{\prime}} h\right)} .
\end{aligned}
$$

One may also show that

$$
a=\frac{1+R R^{\prime}-T T^{\prime}}{2 \sqrt{R R^{\prime}}} .
$$

In case of a symmetric, homogeneous medium, one obviously has $K=K^{\prime}, S=S^{\prime}$, and retrieves the reflectance and transmittance expressions, identical at both sides, given by Kubelka in [2]:

$$
R=R^{\prime}=\frac{1}{a+b \operatorname{coth}(b S h)},
$$

and

$$
T=T^{\prime}=\frac{b}{a \sinh (b S h)+b \cosh (b S h)},
$$

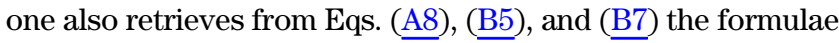
given in [2]]:

$$
R_{\infty}=\frac{1}{a+b}=a-b
$$

and

$$
a=\frac{K+S}{S}=\frac{1+R^{2}-T^{2}}{2 R} .
$$

\section{APPENDIX C: DISTANCE BETWEEN TWO MATRICES}

In Section 6, we present various methods to compute the effective surface coverage of one ink from a printed halftone where this ink is printed at nominal surface coverage 0.25 , 0.5 , or 0.75 on the paper, or on top of the paper coated with a solid layer of primary $v$. The effective surface coverage of the ink is computed as the value that minimizes the difference between the predicted and measured spectra. One method is based on the difference between transfer matrices created from measured and predicted spectra. We detail here how to assess the difference between two matrices by using the concept of matrix norm. One usual norm for a matrix $\mathbf{M}$ is defined in terms of spectral radius of the Gramian matrix $\mathbf{M}^{T} \mathbf{M}$ : 


$$
\|\mathbf{M}\|_{2}=\sqrt{\delta\left(\mathbf{M}^{T} \mathbf{M}\right)}
$$

where symbol $\delta(\mathbf{X})$ denotes the spectral radius of matrix $\mathbf{X}$, i.e., the eigenvalue of $\mathbf{M}$ with highest modulus. The term spectral is related to the spectrum of a matrix, i.e., the set of its eigenvalues, and should not be confused with wavelengths of light.

The effective surface coverage $\tilde{a}_{u / v}$ of the ink $u$ on top of the primary $v$ is fitted as

$$
\tilde{a}_{u / v}=\underset{0 \leq x \leq 1}{\arg \min } \sum_{\lambda \in \text { vis }}\left\|\mathbf{A}_{u / v}^{(m)}(\lambda)-\mathbf{A}_{u / v}^{(x)}(\lambda)\right\|_{2}^{1 / \eta},
$$

where "vis" denotes the visible spectrum of light, $\eta$ is a real number, $\mathbf{A}_{u / v}^{(m)}(\lambda)$ is the matrix created from the measured reflectance and transmittance spectra, and

$$
\mathbf{A}_{u / v}^{(x)}(\lambda)=\mathbf{F} \cdot \frac{1}{\tau_{u / v}^{(x)}}\left(\begin{array}{cc}
1 & -\rho_{u / v}^{\prime(x)} \\
\rho_{u / v}^{(x)} & \tau_{u / v}^{(x)} \tau_{u / v}^{(x)}-\rho_{u / v}^{(x)} \rho_{u / v}^{\prime(x)}
\end{array}\right) \cdot \mathbf{F}^{\prime}
$$

with $\tau_{u / v}^{(x)}, \rho_{u / v}^{(x)}, \rho_{u / v}^{\prime(x)}$, and $\tau_{u / v}^{\prime(x)}$ given by Eq. (33).

We observe in practice that the agreement between the predicted and measured spectra for the four transfer factors is better when the $\eta$ value increases. We can therefore directly consider the following formula, which is the limit of Eq. (C2) as $\eta$ tends to infinity:

$$
\tilde{a}_{u / v}=\underset{0 \leq x \leq 1}{\arg \min }\left(\prod_{\lambda \in \text { vis }}\left\|\mathbf{A}_{u / v}^{(m)}(\lambda)-\mathbf{A}_{u / v}^{(x)}(\lambda)\right\|_{2}\right) .
$$

\section{ACKNOWLEDGMENTS}

This work was performed within the framework of the LABEX MANUTECH-SISE (ANR-10-LABX-0075) of Université de Lyon, within the program "Investissements d'Avenir" (ANR11-IDEX-0007) operated by the French National Research Agency (ANR).

\section{REFERENCES}

1. P. Kubelka and F. Munk, "Ein Beitrag zur Optik der Farbanstriche," Z. Tech. Phys.. 12, 593-601 (1931).

2. P. Kubelka, "New contributions to the optics of intensely lightscattering material, part I," J. Opt. Soc. Am.. 38, 448-457 (1948).

3. P. Kubelka, "New contributions to the optics of intensely lightscattering materials, part II: nonhomogeneous layers," J. Opt. Soc. Am.. 44, 330-335 (1954).

4. G. Kortüm, Reflectance Spectroscopy (Springer Verlag, 1969).

5. J. L. Saunderson, "Calculation of the color pigmented plastics," J. Opt. Soc. Am. 32, 727-736 (1942).

6. F. R. Clapper and J. A. C. Yule, "The effect of multiple internal reflections on the densities of halftone prints on paper," J. Opt. Soc. Am. 43, 600-603 (1953).

7. F. C. Williams and F. R. Clapper, "Multiple internalreflections in photographic color prints,” J. Opt. Soc. Am.. 43, 595-597 (1953).

8. M. Hébert and R. D. Hersch, "Extending the Clapper-Yule model to rough printing supports," J. Opt. Soc. Am. A 22, 1952-1967 (2005).

9. M. Hébert, R. Hersch, and J.-M. Becker, "Compositional reflectance and transmittance model for multilayer specimens," J. Opt. Soc. Am. A 24, 2628-2644 (2007)

10. M. Hébert and J. M. Becker, "Correspondence between continuous and discrete two-flux models for reflectance and transmittance of diffusing layers," J. Opt. A Pure Appl. Opt. 10, 035006 (2008).
11. M. Hébert and J. Machizaud, "Spectral reflectance and transmittance of stacks of nonscattering films printed with halftone colors," J. Opt. Soc. Am. A 29, 2498-2508 (2012).

12. L. Simonot, M. Elias, and E. Charron, "Special visual effect of art-glazes explained by the radiative transfer equation," Appl Opt.. 43, 2580-2587 (2004).

13. H. Pauli and D. Eitel, "Comparison of different theoretical models of multiple scattering for pigmented media," Colour 73, 423-426 (1973).

14. S. Chandrasekhar, Radiative Transfer (Dover, 1960).

15. B. Maheu, J. N. Letouzan, and G. Gouesbet, "Four-flux models to solve the scattering transfer equation in terms of Lorentz-Mie parameters," Appl. Opt. 23, 3353-3362 (1984).

16. J. S. Arney and S. Yamaguchi, "The physics behind the YuleNielsen equation," in PICS 1999: Image Processing, Image Quality, Image Capture, Systems Conference (Society for Imaging Science and Technology, 1999), pp. 381-385.

17. G. Rogers, "A generalized Clapper-Yule model of halftone reflectance," Color Res. Appl. 25, 402-407 (2000).

18. M. Vöge and K. Simon, "The Kubelka-Munk model and Dyck paths," J. Stat. Mech. 2007, P02018 (2007).

19. L. Yang, "Probabilistic spectral model of color halftone incorporating substrate fluorescence and interface reflections," J. Opt Soc. Am. A 27, 2115-2122 (2010).

20. F. Abeles, "La théorie générale des couches minces," J. Phys. Radium 11, 307-309 (1950).

21. P. Emmel, "Physical models for color prediction," in Digital Color Imaging Handbook, G. Sharma and R. Bala, eds. (CRC Press, 2003), pp. 173-238.

22. A. Wolf, B. Terheiden, and R. Brendel, "Light scattering and diffuse light propagation in sintered porous silicon," J. Appl. Phys. 104, 033106 (2008).

23. P. Emmel and R. D. Hersch, "A model for colour prediction of halftoned samples incorporating light scattering and ink spreading," in Proceedings of the 7th IS\&T/SID Color Imaging Conference: Color Science, Systems and Applications (Society for Imaging Science and Technology, 1999), pp. 173-181.

24. M. Hébert and R. D. Hersch, "A reflectance and transmittance model for recto-verso halftone prints," J. Opt. Soc. Am. A 21, 2415-2432 (2006).

25. E. Centurioni, "Generalized matrix method for calculation of internal light energy flux in mixed coherent and incoherent multilayers," Appl. Opt. 44, 7532-7539 (2005).

26. M. C. Troparevsky, A. S. Sabau, A. R. Lupini, and Z. Zhang, "Transfer-matrix formalism for the calculation of optical response in multilayer systems: from coherent to incoherent interference," Opt. Express 18, 24715-24721 (2010).

27. C. C. Katsidis and D. I. Siapkas, "General transfer-matrix method for optical multilayer systems with coherent, partially coherent, and incoherent interference," Appl. Opt. 41, 3978-3987 (2002).

28. C. L. Mitsas and D. I. Siapkas, "Generalized matrix method for analysis of coherent and incoherent reflectance and transmittance of multilayer structures with rough surfaces, interfaces, and finite substrates," Appl. Opt. 34, 1678-1683 (1995).

29. M. Born and E. Wolf, Principle of Optics, 7th ed. (Pergamon, 1999).

30. L. Simonot, M. Hébert, and R. Hersch, "Extension of the Williams-Clapper model to stacked nondiffusing colored layers with different refractive indices," J. Opt. Soc. Am. A 23, 1432-1441 (2006).

31. D. B. Judd, "Fresnel reflection of diffusely incident light," J. Res Natl. Bur. Stand.. 29, 329-332 (1942).

32. F. E. Nicodemus, J. C. Richmond, J. J. Hsia, I. W. Ginsberg, and T. Limperis, Geometrical Considerations and Nomenclature for Reflectance, Vol. 160 of NBS Monograph (National Bureau of Standards, 1977), p. 52.

33. CIE, "Colorimetry," CIE Technical Report 15, 3rd ed. (CIE, 2004).

34. H. E. J. Neugebauer, "Die theoretischen Grundlagen des Mehrfarbendrucks," Z. Wiss. Photogr. 36, 36-73 (1937) [Color Res. Appl. 30, 322-331 (2005)].

35. M. Hébert and R. D. Hersch, "Reflectance and transmittance model for recto-verso halftone prints: spectral predictions with multi-ink halftones," J. Opt. Soc. Am. A 26, 356-364 (2009). 
36. R. D. Hersch and F. Crété, "Improving the Yule-Nielsen modified spectral Neugebauer model by dot surface coverages depending on the ink superposition conditions," Proc. SPIE 5667, 434-445 (2005).

37. M. Hébert and R. D. Hersch, "Review of spectral reflectance models for halftone prints: principles, calibration, and prediction accuracy," Color Res. Appl., doi:10.1002/col.21907 (to be published).

38. G. Wyszecki and W. S. Stiles, Color science: concepts and methods, in Quantitative Data and Formulae, 2nd ed. (Wiley, 1982).
39. M. Hébert and R. D. Hersch, "Classical print reflection models: a radiometric approach,” J. Imaging Sci. Technol. 48, 363-374 (2004).

40. M. Hébert, R. Hersch, and L. Simonot, "Spectral prediction model for piles of nonscattering sheets," J. Opt. Soc. Am. A 25, 2066-2077 (2008).

41. J. Machizaud and M. Hébert, "Spectral reflectance and transmittance prediction model for stacked transparency and paper both printed with halftone colors," J. Opt. Soc. Am. A 29, 1537-1548 (2012).

42. G. Strang, Applied Mathematics (MIT, 1986). 